\title{
Osteointegration Characterization of Additive Manufactured Porous Titanium Scaffold Based on Microhardness and Ca/P Ratio
}

\section{M.V. Gilev ${ }^{1,2}$, Yu.N. Loginov ${ }^{2,3}$, I.A. Naschetnikova ${ }^{2}$, M.S. Karabanalov ${ }^{2}$, and S.l. Stepanov ${ }^{2}$}

1Ural State Medical University, 3 Repina str., Ekaterinburg, 620014, Russia

${ }^{2}$ Ural Federal University named after the first President of Russia B.N.Yeltsin, 19 Mira str., Ekaterinburg, 620002, Russia

${ }^{3}$ M.N. Miheev Institute of Metal Physics of Ural Branch of Russian Academy of Sciences, $18 \mathrm{~S}$. Kovalevskaya Street, Yekaterinburg, Russia, 620137

\section{Abstract}

Additive technologies are getting widely used in orthopedics due to the opportunity of full modelling of complex cellular structures and producing personalized implants. Titanium and its alloys are still the main material used for metallic implants

Corresponding Author:

I.A. Naschetnikova

naschetnikova@mail.ru

Received: 25 February 2019

Accepted: 9 April 2019

Published: 15 April 2019

Publishing services provided by Knowledge E

(c) M.V. Gilev et al. This article is distributed under the terms of the Creative Commons

Attribution License, which permits unrestricted use and redistribution provided that the original author and source are credited.

Selection and Peer-review under the responsibility of The Ural school-seminar of metal scientists-young researchers Conference Committee. manufacturing. In this study microhardness and $\mathrm{Ca} / \mathrm{P}$ ratio of bone tissue surrounding the implant were investigated. By means of ABAQUS software titanium porous structure implants were designed. Their biomechanical properties were close to biomechanical properties of trabecular bone tissue. Direct metal laser sintering (DMLS) was used to manufacture implants from Ti-6Al-4V titanium alloy. They were augmented in trabecular bone defects of Chinchilla rabbits for 6, 12 and 25 weeks. The samples were examined by scanning electron microscopy (SEM) in a FEG SEM ZEISS CrossBeam AURIGA and the $\mathrm{Ca} / \mathrm{P}$ ratio was calculated using the accompanying Oxford Inca software. Vickers hardness test method was performed for microhardness. It was shown that there was a significant increase in both mineral and mechanical properties of bone samples with an increase of observation period. The results found in this study illustrate a distinct relationship between the mineralization parameter of bone tissue and its microhardness $\left(r=0.808, r^{2}=0.65\right)$.

Keywords: Scaffold, Microhardness, Titanium, Ca/P ratio, Bone defect, Osteointegration, Additive manufacturing

\section{Introduction}

Various materials and techniques have been developed to facilitate bone-healing process and reduce its healing period. Tissue engineering of bone scaffolds may offer a different variety to native autoscaffolds, which are in short supply. Metallic biomaterials are widely used in bone surgery for different approaches. Titanium and its alloys are preferred as implant materials, as they are highly biocompatible, osteoconductive and 
resistant to corrosion [1]. Young's modulus as well as tensile strength, ductility, fatigue life, fretting fatigue life, wear properties, functionalities, etc., should be adjusted to the levels that are suitable for structural biomaterials used in implants that replace hard tissue [2].

Many studies promote the fabrication of scaffolds consisting of a viable porous bonelike metal. Introduction of porosity and pore interconnectivity improves mechanical fixation and osteointegration by allowing extensive body fluid transport through the porous implant. This can provoke bone tissue ingrowth, consequently leading to the development of a stable interface between the scaffold and host tissue [3]. When an implant is populated with osteogenic cells, these cells not only migrate on the surface of the implant but also inside the pores of the implant [4]. So the introduction into operative trauma and orthopedics clinical practice porous titanium augments obtained based on additive prototyping undoubtedly requires an active study of the fundamental principles of bone tissue regeneration.

Advantages of the manufacturing process have led to the evaluation of porous titanium as the bone-like base material [5]. For manufacturing the porous titanium implants, such methods as selective laser melting, electron beam melting and direct metal laser sintering have proven to be relevant methods as they offer the opportunity to produce patient-individual implants for different approaches quickly and cost-efficiently. The porous surfaces of DMLS titanium parts have been demonstrated to be favorable for cell adhesion, migration and ingrowth and these properties result in a strong boneimplant contact [6].

Mechanical properties are especially important in scaffolds for hard and ductile tissues such as bone because the scaffolds must also interact with their physiological surroundings to transmit mechanical signals to cells and regulate cell behavior (i.e., differentiation, motility, and contractility). The stiffness of scaffold can have effects at a transcriptional level, determining whether stem cells make the decision to become cells as functionally diverse as osteoblasts [7]. Mechanical mismatch of implant and bone defects frequently leads to loosening of the implant. The higher Young's modulus of titanium compared to that of cortical or trabecular bone resulting in stress shielding is of major concern [8-10].

Due to advantages of the titanium structures produced by DMLS, we designed and produced a porous augment for experimental estimation of its osteointegration properties with microindentation and energy dispersive $\mathrm{X}$-ray microanalysis. 


\section{Material and Methods}

The porous titanium implant (PTI) was an experimental medical device and consisted of cubic samples with the side of $5 \mathrm{~mm}$, which were produced by direct metal laser sintering (DMLS) on an EOSINT 280. As a raw material, a powder was used from the $(\alpha+\beta)$-titanium alloy Ti-6Al-4V Eli. The dimensions and geometry of the unit cell of the porous structure provided open-porosity in the samples under study of the order of 55\%.

Preliminary finite element calculations in the ABAQUS software have shown the importance of adequate design of the internal architecture of a cellular material. As a result, the cellular structure was used as the base architecture for volumetric modeling by the finite element method, the unit cell of which was a diamond structure, which consisted of cylinders oriented in space at an angle of $109^{\circ} 28^{\prime}$ to each other. In the software package Solid Works, 3D models of a unit cell with different pore fractions were constructed. Samples were designed in the form of a parallelepiped with open porosity. The pore size was $680 \mu \mathrm{m}$, the spacer size varied in the interval 400-1300 $\mu \mathrm{m}$, so the relative porosity of the sample was set in the interval $50 . . .80 \%$. Ti-6Al-4V titanium alloy was used as the model material, as the most commonly used alloy for manufacturing medical implants.

Twenty-four 6 months female Shinshila rabbits weighing $(3.11 \pm 0.31) \mathrm{kg}$ were used. The animals were kept in individual cages $\left(22^{\circ} \mathrm{C}, 55 \%\right.$ humidity, and a 12 -h light/dark cycle) and were fed and watered ad libitum. The study protocol was approved by the Ethics Committee of Ural Medical University (December 2016, protocol №10). These rabbits were randomly divided into two groups (test and control groups). Operation was performed under general anesthesia - intramuscular xylazine $20 \mathrm{mg}-1 \mathrm{ml}-0.2 \mathrm{ml} / \mathrm{kg}$ and tiletamine $-6 \mathrm{mg} / \mathrm{kg}$. To potentiate anesthesia, $0.25 \%$ of novocaine was used, which was also used with hydraulic tissue dissection property. Augmentation procedure was performed bilaterally. Both shins were shaved and washed with $10 \%$ povidone iodine solution.

Surgical access was performed to the medial tibia condyle by soft tissues sharp dissection; the periosteum was displaced in the distal direction. The anatomical reference point for further action was the general tendon fixation of pes anserinus superficialis. With a diamond metal cutting disc we produced a quadruple spacing $(7 \times 8 \mathrm{~mm})$ in the medial condyle of the tibia before the anatomical formation above the depth of the cortical plate of the bone without penetrating the cavity of the knee joint, after which the desired rectangular part of the bone was extracted. A dynamic force displacement (using a hammer and an impactor) of the proximal condyle fragment in the distal direction 
was performed to make a defect for modelling the intraarticular impressional fracture. To manage such fracture an open reduction was done. Rabbits from test group were augmented with PTI by press fit type, controls were not augmented. The periosteum, muscle fascia, and skin were then sutured in separate layers. An analgesic (1 mg/kg tramadol) and an antibiotic ( $25 \mathrm{mg} / \mathrm{kg}$ cefazolin) were administered intramuscularly preoperatively and twice per day for three postoperative days.

Five rabbits of test group and three rabbits of control were euthanized via an intravenous tiletamine overdose at 6,12 and 25 weeks after the scaffold placement. The proximal tibias were fixed in $10 \%$ formalin.

Bone samples for further research after cooling with liquid nitrogen to prevent bone structure thermal destruction and to reduce the bone-implant viscosity were sliced (5 $\mathrm{mm}$ distally from the joint surface to lay the cut at the middle of PTI) with diamond metal cutting disc. Further, grinding was carried out using an abrasive wheel and final polishing with a colloidal silica suspension. After that, the samples were degreased and cleaned in an ultrasonic bath in acetone. The adhesive glue was applied in a thin layer and the sample was stabilized using digital pressure.

Vickers Hardness (VHN) of the bone was assessed by using a microhardness dynamic indenter (PMT-3 microhardness tester, Russia). Ten continuous indentations were made around each critical zones (less than $500 \mu \mathrm{m}$ ). The hardness is defined as the test force divided by the apparent area of the indentation at maximal force. The holding time was 15 seconds, and the applied load was $5 \mathrm{~N}$. The diagonals of the prints were measured at a 200-fold magnification, and the Vickers microhardness value was calculated by the formula:

$$
V H=\frac{0,189 \times F}{d^{2}}
$$

where $\mathrm{F}$ is the applied load, [N]; $d$ is the length of the diagonal, [mm].

The same samples were examined by scanning electron microscopy (SEM) in a FEG SEM ZEISS CrossBeam AURIGA. The $\mathrm{Ca} / \mathrm{P}$ ratio was calculated using the accompanying Oxford Inca software the same critical zones. Reference standards and synthetic pure samples with similar $\mathrm{Ca} / \mathrm{P}$ ratio (tricalcium phosphate, $\mathrm{Ca}_{3}\left(\mathrm{PO}_{4}\right)_{2}$, and hydroxyapatite) were used for calibration. Prior to each session, the smooth surface of all pellets was coated with a conductive carbon layer (Fig. 1).

STATA software was utilized for the statistical analysis of the results. Data are reported as median with interquartile range (IQR) and confidence intervals ( $\mathrm{Cl}$ ). In the analysis of the data, parametric (Student's test) and non-parametric methods (Wilcoxon test, Kruskal-Wallis) and independent (Mann-Whitney test) variants were used, as well as the 
Shapiro-Wilk test to verify the normality of distributions. The degree of interconnection of traits was estimated using Pearson correlation coefficient and nonparametric Spearman coefficient. Statistical significance was considered at $p<0.05$.

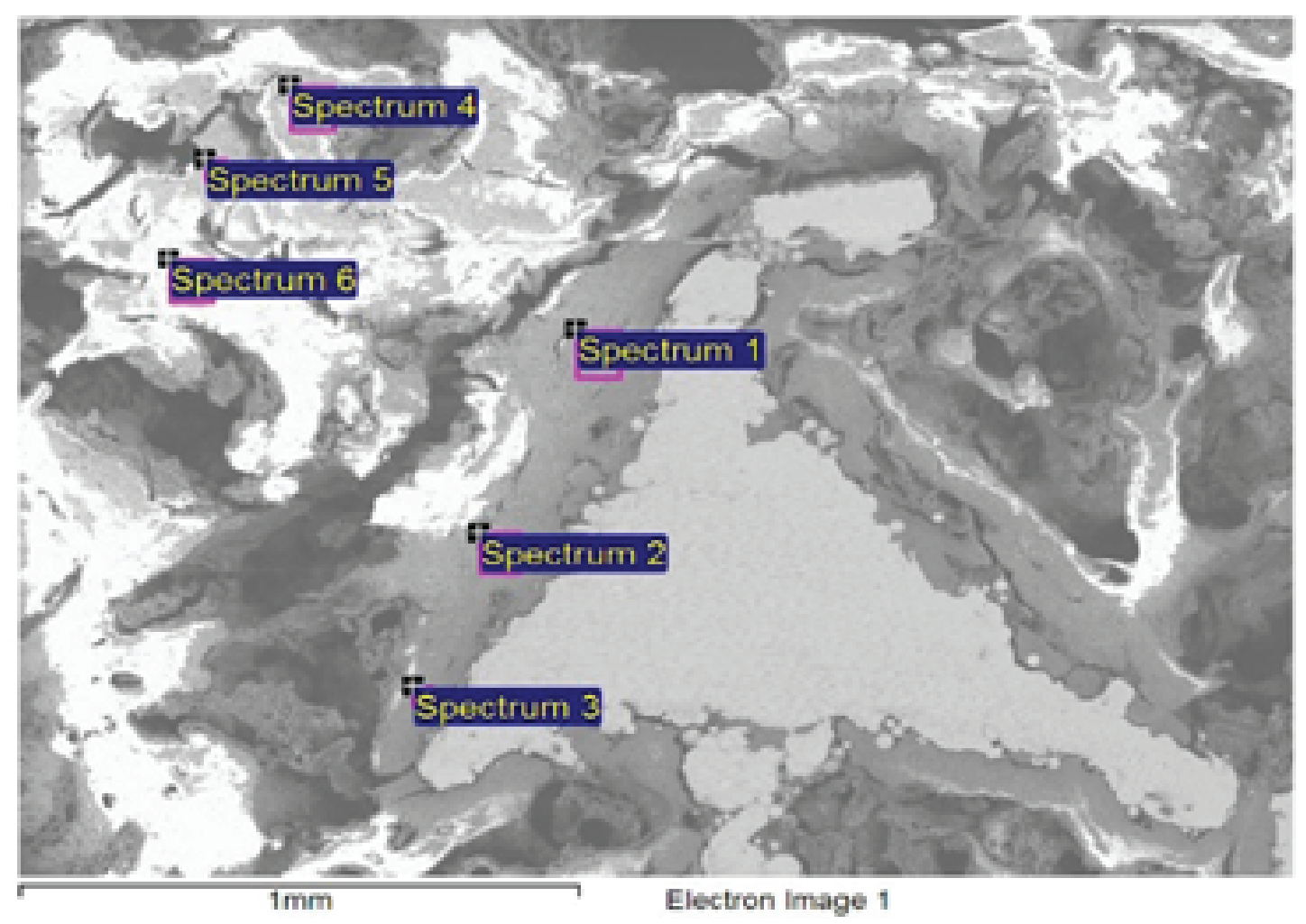

Figure 1: Photomicrograph of trabecular bone tissue site with an augmented porous titanium implant.

\section{Results}

No operative or postoperative complications were encountered. All of the rabbits tolerated surgery well and survived until the final experimental time. No wound opening or infections were observed. At sacrifice the macroscopic evaluation revealed maintenance of correct position of sample in the defect site.

There was a significant increase in both mineral and mechanical properties of bone samples in test group in all times as compared to control group (Table 1).

The Pearson correlation test between the microhardness parameter and the $\mathrm{Ca} / \mathrm{P}$ ratio revealed the strong relationship $\left(r=0.808, r^{2}=0.65\right)$. 
TABLE 1: Ca/P ratio and microhardness of test and controls groups (median, 95\% Confidence interval).

\begin{tabular}{l|c|c|c|c|}
\hline \multirow{2}{*}{ Time of augmentation } & \multicolumn{2}{|c|}{ Ca/P ratio } & \multicolumn{2}{c|}{ Microhardness [HV] } \\
\hline & Test group & Control & Test group & Control \\
\hline 6 weeks & $1.42(1.28-1.64)^{*}$ & $1.94(1.92-1.98)$ & $66(63-69)^{*}$ & $87(74-91)$ \\
\hline 12 weeks & $1.54(1.16-1.89)^{*}$ & $1.95(1.91-1.98)$ & $82(74-88)$ & $88(84-91)$ \\
\hline 25 weeks & $2.03(1.74-2.17)$ & $1.94(1.91-1.98)$ & $99(94-102)^{*}$ & $87(84-91)$ \\
\hline${ }^{*} \mathrm{p}<0.05$ & & & &
\end{tabular}

\section{Discussion}

$\mathrm{Ca}$ and $\mathrm{P}$ are the main elements of the bone mineral matrix. The relative content of $\mathrm{Ca}$ and $\mathrm{P}$, however, is critical for sustaining mineral homeostasis and bone metabolism and their co-dependence is evident for bone growth and development [11]. It is therefore a suitable biomarker for the assessment of bone mineral homeostasis, which an energydispersive (EDX) analysis is physically able to measure from relative mass variations of $\mathrm{Ca}$ and $\mathrm{P}$ in different bone samples. Kourkoumelis $\mathrm{N}$. et all evaluated the $\mathrm{Ca} / \mathrm{P}$ ratio at different sites of normal and osteoporotic rabbit bones using two spectroscopic techniques: auger electron spectroscopy and EDX. They found $\mathrm{Ca} / \mathrm{P}$ ratio of normal trabecular bone as $1.88 \pm 0.04$ (mean $\pm S D$ ). They also came to conclusion that there is no significant difference between samples from different genders or among cortical bone sites ( $\mathrm{Ca} / \mathrm{P}$ ratio for rear tibia was $2.14 \pm 0.04)$ [12]. Other data were obtained in the study of Sobczak A. et all. The calcium to phosphorus ratio in the animal bony samples had value of 1.67 and amounted to 1.70 [13]. According to Zuo Q. et all Ca/P ratio of human subchondral bone was $1.52 \pm 0.04$ [14].

In our study, the $\mathrm{Ca} / \mathrm{P}$ ratio in perifocal zone at 6 weeks of implantation was 1.42 (1.28-1.64) that was 1.37 times lower comparing controls. This may indicate the presence of immature bone tissue, which undergoes mineralization processes. At 25 weeks of implantation ratio was 1.42 (1.28-1.64), that was 1.05 times higher comparing controls ( $p$ $>0.05$ ), which may indicate the mineralization processes completion and the presence of mature lamellar bone tissue, which in its characteristics does not differ from the control group.

Another important characteristic of bone tissue in terms of mechanical properties is the Vickers microhardness. Soares P.B. et all studied the Vicker's hardness and elastic modulus surround cortical bone to dental implant in different regions. Ten cylindrical implants with morse taper interface (Titamax CM, Neodent; $3.5 \mathrm{~mm}$ diameter and 7 $\mathrm{mm}$ a height) were inserted in rabbit tibia. Mean and standard deviation of Vickers microhardness was $120.1 \pm 43.7$ [15]. According to Stea S. et all who evaluated bone 
microhardness at the interface with hydroxyapatite-coated stainless-steel pins as test group and uncoated pins were implanted as controls. Pins were transversally inserted into the diaphyses of sheep tibiae and were loaded in for 6 weeks. The mean hardness at the interface with the pins was 56.9 Vickers, whereas at the interface with the uncoated pins it was 62.2. Authors came to conclusions that, 6 weeks postsurgery, the bone growing into the threadings of a loaded screwed implant reached maturity at a degree lower than that of the host both uncoated and coated implants [16]. Giavaresi G. et all evaluated bone microhardness in implants perifocal zone inserted in sheep femoral cortical bone at different times to compare the in vivo osseointegration of titanium screws. It was concluded that titanium implants showed the lowest value for the inner thread area at 8 weeks $(49.8 \pm 3.8 \mathrm{HV})$. These findings confirm that osseointegration may be accelerated by adequate surface roughness and bioactive ceramic coating such as Ca-P [17].

In our study, the Vickers microhardness in perifocal zone at 6 weeks of implantation was 66 (63-69) HV that was 1.32 times lower comparing controls ( $p<0.05)$. At 25 weeks of implantation Vickers microhardness was 99 (94-102) HV, that was 1.14 times higher comparing controls $(p<0.05$ ), which may indicate the maturation processes completion, characteristics of which to some extent exceed the control group. In our study, with an increase in the observation period, a distinct relationship was observed between the mineralization parameter of bone tissue and its microhardness $\left(r=0.808, r^{2}=0.65\right)$, so it can be assumed that porous titanium implants have a good integration properties into the trabecular bone defect.

\section{Conclusion}

The results of this study suggest that porous titanium implants are effective in trabecular bone augmentation when employed as a bone substitute as evidenced through $\mathrm{Ca} / \mathrm{P}$ ratio and microhardness estimation. Though animal models cannot be directly translated to clinical application, investigation into the use of porous titanium implants in a clinical setting may be appropriate to further characterize its efficacy.

\section{Acknowledgements}

We hereby acknowledge the support of the Ministry of Science and Education of the Russian Federation, in accordance to the decree of the Government of April 9, 2010, №218, project number 03.G25.31.0234. 
The work was carried out using the laboratory equipment "Structural methods of analysis and properties of materials and nanomaterials" of the Center of Ural Federal University.

\section{References}

[1] J.P. Li, S.H. Li, C.A. Van Blitterswijk, K. de Groot. Cancellous bone from porous Ti6AI4V by multiple coating technique. J. Mater. Sci. Mater. Med. 17 (2006) 179-185.

[2] M. Niinomi. Mechanical biocompatibilities of titanium alloys for biomedical applications. J. Mech. Behav. Biomed. Mater. 1 (2008) 30-42.

[3] B. Dabrowski, W. Swieszkowski, D. Godlinski, K.J. Kurzydlowski. Highly porous titanium scaffolds for orthopaedic applications. J. Biomed. Mater. Res. B Appl. Biomater. 95 (2010) 53-61.

[4] J. Matena et al. Characterisation of Cell Growth on Titanium Scaffolds Made by Selective Laser Melting for Tissue Engineering. Biomed. Tech. 58 (2013).

[5] A.B. Nover et al. Porous titanium bases for osteochondral tissue engineering. Acta Biomater. 27 (2015) 286-293.

[6] W. Peng, L. Xu, J. You, L. Fang, Q. Zhang. Selective laser melting of titanium alloy enables osseointegration of porous multi-rooted implants in a rabbit model. Biomed. Eng. 15 (2016).

[7] M. Tamaddon, S. Samizadeh, L. Wang, G. Blunn, C. Liu. Intrinsic Osteoinductivity of Porous Titanium Scaffold for Bone Tissue Engineering. Int. J. Biomater. (2017).

[8] K. Zhu, C. Li, Z. Zhu, C.S. Liu. Measurement of the dynamic Young's modulus of porous titanium and Ti6Al4V. J. Mater. Sci. 42 (2007) 7348-7353.

[9] M.A. Lopez-Heredia et al. Rapid prototyped porous titanium coated with calcium phosphate as a scaffold for bone tissue engineering. Biomaterials. 29 (2008) 26082615.

[10] G.E. Ryan, A.S. Pandit, D.P. Apatsidis. Porous titanium scaffolds fabricated using a rapid prototyping and powder metallurgy technique. Biomaterials. 29 (2008) 36253635.

[11] R. Shapiro, R.P. Heaney. Co-dependence of calcium and phosphorus for growth and bone development under conditions of varying deficiency. Bone. 32 (2003) 532540.

[12] N. Kourkoumelis, I. Balatsoukas, M. Tzaphlidou. Ca/P concentration ratio at different sites of normal and osteoporotic rabbit bones evaluated by Auger and energy dispersive X-ray spectroscopy. J. Biol. Phys. 38 (2012) 279-291. 
[13] A. Sobczak, Z. Kowalski, Z. Wzorek. Preparation of hydroxyapatite from animal bones. Acta Bioeng Biomech. 11 (2009) 23-28.

[14] Q. Zuo et al. Characterization of nano-structural and nano-mechanical properties of osteoarthritic subchondral bone. BMC Musculoskelet. Disord. 17 (2016) 367-379.

[15] P.B. Soares et al. Measurement of Elastic Modulus and Vickers Hardness of Surround Bone Implant Using Dynamic Microindentation - Parameters Definition. Braz. Dent. J. 25 (2014) 385-390.

[16] S. Stea et al. Microhardness of bone at the interface with ceramic-coated metal implants. J. Biomed. Mater. Res. 2 (1995) 695-699.

[17] G. Giavaresi et al. Histomorphometric and microhardness assessments of sheep cortical bone surrounding titanium implants with different surface treatments. J. Biomed. Mater. Res. A. 67 (2003) 112-120. 